excitation probabilities. In our approach, these excitations come about in two ways: the first is through the direct coupling of the spin vector to the quantized electromagnetic field; the other is through fluctuations in the particle orbit. It is the vertical fluctuations (in the direction of the magnetic field) which induce spin transitions. Through the Heisenberg equations of motion, these fluctuations in turn can be related to the quantum fluctuations in the electromagnetic fields. The field fluctuations build up the fluctuations in the orbit and radiation damping prevents them from growing indefinitely.

The part of the fluctuations in the vector $\vec{\omega}$, which is responsible for the spin excitations, can then be written in the simple form

$$
\delta \vec{\omega}=\frac{e}{2 m c}\left[g B_{\mathrm{q}}^{\prime}+\left(2 e_{\mathrm{r}}+f(\mathrm{~g})\right) E_{\mathrm{qz}}^{\prime}\right]
$$

where $B_{\mathrm{q}}^{\prime}$ and $E_{\mathrm{qz}}^{\prime}$ are the free quantum fields in the rest frame of the electron and $e_{r}$ is a unit vector in the radial direction. The resonance factor $f(g)$ is proportional to $g$-2: being a function of $\gamma$ it is essentially equal to 0 except in a narrow interval where there is resonance between the vertical fluctuations and the spin motion. The transition probabilities between the spin levels now are determined by correlation functions of along the classical orbit. For $\boldsymbol{f}(g)=0$, the result found in this way for the transverse polarization of the electrons agrees with the earlier result [5].

An interesting additional observation concerns the resonance factor $f(g)$. In our approach one sees that the resonance does not have a purely depolarizing effect. As a function of $\gamma$, the polarization close to the point of resonance initially decreases, from 0.92 to -0.17 , but then increases to 0.99 before finally decreasing again to 0.92 . It is thus possible, at least in principle, to exceed the "maximum" value 0.92 close to the resonance.

\section{Conclusions}

Returning to the question of the relation between the polarization effect and the Unruh effect one sees, as already stated, that they are of a similar nature. Transitions between spin levels are caused by quantum fluctuations of the vacuum fields along the electron orbit, in the same way as field fluctuations induce transitions in a linearly accelerated detector. In this sense, both the electrons and the detector are heated by the accelerating motion through a vacuum.

However, there is also an essential difference between the two cases. For uniform linear acceleration, the excitation spectrum has a universal thermal character. This is not the case for the "circular Unruh effect" where the excitation spectrum depends on characteristics of the detector. Using circulating electrons, the effects of Thomas precession are important and fluctuations in the orbit cannot be neglected.

\section{ADDITIONAL REFERENCES}

[1] Fulling S.A., Phys. Rev. D 7 (1973) 2850. [2] Hawking S., Nature 248 (1974) 30; Commun. Math. Phys. 43 (1975) 199.

[3] Unruh W.G., Phys. Rev. D 14 (1976) 870.

[4] Sokolov A.A. and Ternov I.M., Dokl. Akad. Nauk. SSR 153 (1963) 1052.

[5] Derbenev Ya.S. and Kondratenko A.M. Zh. Exp. Teor. Fiz. 64 (1973) 1918; Jackson J.D., Rev. Mod. Phys. 48 (1976) 417.

\section{John Bell Symposium}

A Symposium on Quantum Physics, in memory of John Stewart Bell who died on 1 October 1990, will be held at CERN on 2-3 May. The programme includes talks by $A$. Aspect, H. Rauch, A.J. Leggett, K. Gottfried, A. Shimony, G.C. Ghirardi, J. Leinaas and R. Jackiw. Further information: Jeanne Rostant, Theory Division, CERN, CH-1211 Genève 23 (Tel./Fax: +41 (22) $7674222 / 39$ 14; Bitnet: rostant@cernvm.cern.ch).

\title{
World Lab Fosters Third World Excellence
}

Establishing a "new equilibrium" in the distribution of knowledge between east and west, north and south can provide a "new way out" for undeveloped countries by allowing the creation of scientifically based cultures which have the capacity to generate wealth from limited material resources. But one has to act directly because if one "applies European logic", this process would never have started even in our "corner of the planet". Action must also span all disciplines since "only a strong civilization creates science". Most important of all, experience in creating the great laboratories of Europe implies that solutions will mostly spring from initiatives by individuals.

These remarks by Professor Antonino Zichichi help one understand the principles which guide the International Centre for Science Culture (ICSC), better known as the World Laboratory. Founded by Professor Zichichi, its President, in 1986, the World Lab works to extend the Erice heritage in improving the training of, and opportunities for, scientists based in poorer countries. Erice is the home of the Ettore Majorana Centre for Scientific Culture that celebrated its 25th Anniversary last year with the first award of the
Science for Peace Prize (see Europhysics News 21 (1990) 219). Housed in two converted monasteries and a former convent in the famous medieval hill-top town, the Centre has hosted some 100 schools and 480 courses attended by 44000 participants from 103 countries, a great demonstration of "international collaboration without ideological position". Erice also gives its name to the world renowned Statement written by P.A.M. Dirac, P.L. Kapitza and A. Zichichi whose 10000 signatories declare themselves morally bound to work purely in the interests of science - a "new rôle for science".

\section{World Laboratory}

The World Lab builds upon the Erice principles through collaborative projects in science and technology with existing institutions. The Lab's operating budget comes largely from Italy, the USSR and China, but this does not imply that it is just another funding agency. Each beneficiary country in fact handles local projects, thus requiring only a very small administrative team at the headquarters in Lausanne, Switzerland.

The Erice experience also provided a wealth of essential experience and con-

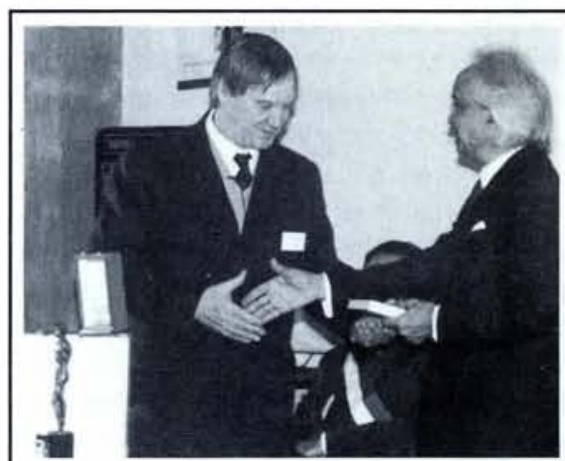

Professor S.P. Kapitza (left) receiving on behalf of his father Academician P.L. Kapitza the first Scienza per la Pace Prize from Professor A. Zichichi last November in Erice, Sicily.

The other winners were P.A.M. Dirac and A.D. Sacharov, E. Teller and V.F. Weisskopf.

tacts so that distinguished colleagues can help identify unique projects. The result is that Professor T.D. Lee of Columbia University was instrumental in setting up in 1986, Beijing's Center for Advanced Science and Technology of which he is the Director. The Center is a branch laboratory established with governmental agreement that ensures independence and effectiveness in running projects in China. It employs 50 scientists and 30 students and, aside from research in high energy 
physics, materials science and superconductivity (an "essential blend of big and small science" ${ }^{\prime \prime}$ ), runs nationwide workshops to provide scientists trained overseas with an attractive support environment. A similar operation now exists in the USSR stemming from a collaboration that started in 1987.

Other eminent collaborators in physics include Professor Abdus Salam who is helping to set up a Centre in Pakistan, and Professor Sam Ting of MIT who leads a World Lab project in high energy physics together with Professor Zichichi from CERN. Professor Kay Siegbahn, Uppsala's Nobel Laureate, is responsible for a project in Sweden concerned with the electron spectroscopy of materials.

\section{Fellows and Scholars}

The World Lab awards Fellowships to scientists from the third world for advanced training and research in industrialized countries. They are expected to return home, where they will be encouraged with a "delta" addition to salaries and help to retain their association with the project in which they participated.

Another key component is the World Laboratory Scholarship programme. Directed by Professor Zichichi, it aims to place the world's 1000 best post-doctoral brains in an almost traditional teacher-student situation with outstanding scientists in advanced countries. Moreover, research should focus on original topics that emphasize third world issues. Some 100 scholarships have been awarded for 1-2 year placements in North American, Japanese and European universities, and exceptional first-year performance can lead to participation in a new World Lab project.

It is difficult to do justice to the depth and spread of the World Lab's projects both inside and outside physics. However, one should mention involvement with the Gran Sasso Laboratory (Europhysics News 21 (1990) 123); the vision of a World Seismology Network and eventually a network for monitoring the earth in real-time; the Yellow River Project in China which aims to understand the river's flooding with the ultimate dream of a real-time warning system; and a food improvement programme (also in China) involving the planting and harvesting of special fruit trees. There are also medical projects in Africa for AIDS and other diseases under the direction of Professors Gallo and Parenzan. In each case, the Laboratory tackles all aspects of a problem with a comprehensive, integrated approach. The bottom line of course is that fostering third world collaboration with the very best in science and technology will help allow third world scientists to learn, cope with, produce and compete with the very best, for only then will they be able to "catalyze" the essential cultural developments in their own countries.

\section{Centre de Physique des Particules de Marseille}

\section{UNIVERSITÉ D'AIX - MARSEILLE II}

In the course of an expansion plan, the Center of Particle Physics of Marseille is expecting to recruit at a senior level, corresponding to a full professor position. The laboratory is currently developing an LHC-SSC physics programme.

At the present time we are involved in LEP, CP LEAR, neutrino oscillation experiments and detector $R$. and $D$. In manpower, the laboratory plans to expand from 65 to around 100 within 2 or 3 years: it will start constructing a new building this year.

\section{Further information J.J. Aubert}

can be obtained from: Centre de Physique des Particules de Marseille

Faculté des Sciences Luminy, Case 907

163 avenue de Luminy, F-13288 Marseille Cédex 09

Tel. +33 () 91418307

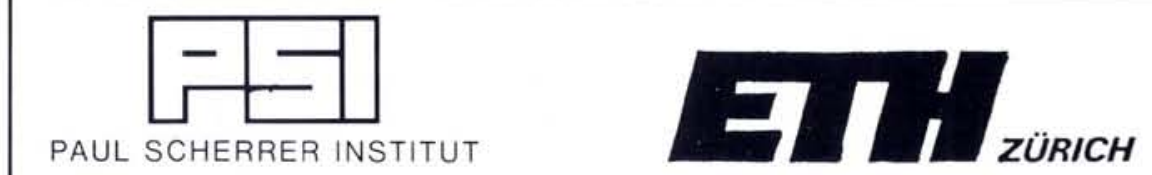

A research team of the Paul Scherrer Institute in Villigen/Würenlingen and the Institute for Intermediate Energy Physics of the Swiss Federal Institute of Technology (ETH) Zürich is engaged in accelerator mass spectroscopy and materials science. Owing to a retirement we are looking for a

\section{Nuclear Physicist and Leader of the Research Group}

The group disposes of one of the leading experimental installations based on a Tandem-Van de Graaff Accelerator and has an excellent scientific reputation in dating, ion implantation and thin-layer activation.

Applicants should have experience in nuclear experimental techniques and ion beam applications. In addition to leading and further developing the ongoing projects of accelerator mass spectroscopy, the candidate will be encouraged to persue innovative new applications of nuclear physics.

We also expect an interest and commitment to teaching in nuclear or accelerator physics at a graduate level.

Candidates should send their resume, a list of publications and the names and addresses of three referees not later than May 31, 1991 to:

The Chairman of the Institute of Intermediate Energy Physics

of the Swiss Federal Institute of Technology

Professor R. Eichler

Paul Scherrer Institute PSI, CH-5232 Villigen (PSI), Switzerland.

\section{UNIVERSITY OF BERNE}

The University of Berne invites applications for an open

\section{Full-Time Professorship}

in the Division of Earth System Science of the Physics Institute (Physikalisches Institut). The appointee is expected to be Head of the Division and to lead its research programme. The position will be available from 1 October 1992.

Applications from established scientists, willing to participate at all levels in the teaching duties of the Physics Institute, should be sent no later than 30 September 1991 to:

Erziehungsdirektion des Kantons Bern, Amt für Hochschulwesen $(1600.51 / 90)$, Sulgeneckstrasse 70, CH-3005 Berne.

In addition to the ususal documentation (detailed curriculum vitae, publication record) an outline of the envisaged research programme should be submitted.

For enquiries contact:

Prof. H. Debrunner, Managing Director,

Physikalisches Institut,

Sidlerstrasse 5, CH-3012 Berne Tel. +41 (31) 654051 